\title{
Optimización de un néctar de camu camu (Myrciaria dubia) utilizando métodología de superficie de
} respuesta

\section{RESUMEN}

Se optimizó la formulación del néctar de camu camu (Myrciaria dubia) empleando Metodología de Superficie de Respuesta (MRS). Las variables fueron: sacarosa y agua y la variable respuesta: la aceptación general. La metodología se llevó a cabo en tres etapas: Screening y Escalamiento con un Diseño Factorial de $2^{k}$ más puntos centrales con 9 tratamientos y una Optimización final con un Diseño Compuesto Central de 13 tratamientos. Los niveles óptimos de las variables fueron: sacarosa $(79,95 \mathrm{~g})$ y agua $(301,22 \mathrm{~g})$. Se realizó un análisis sensorial y pruebas de estabilidad con un tiempo de 80 días de vida útil.

Palabras clave: camu camu, néctar, optimización, superficie de respuesta

OPTIMIZING A CAMU CAMU NECTAR (MYRCIARIA DUBIA) USING RESPONSE SURFACE METHODOLOGY

\section{ABSTRAC}

To optimize a formulation of camu camu (Myrciaria dubia) nectar, a Response surface methodology (RSM) was used. The variables were: sacarose and water and the response was the general acceptability. The methodology employed three stages: Screening and Path of Steepest Ascent with a $2^{\mathrm{k}}$ Factorial design and centrals points with 9 treatments each one and a final optimization with a Central Composite Rotatable design with 13 treatments. The optimal levels of the variables were obtained: sacarose $(79,95 \mathrm{~g})$ and water $(301,22 \mathrm{~g})$. Sensory analysis and stability test with a time of shelf life of 80 days were performed.

Keywords: camu camu, nectar, optimization, response surface

\section{INTRODUCCIÓN}

Uno de los aspectos más importantes para desarrollar un producto es la implementación de técnicas que minimicen el costo, reduciendo el número de formulaciones experimentales para estudiar una característica particular del producto. La metodología de superficie de respuesta (MSR), es una técnica estadística útil para la investigación de procesos complejos y ha sido ampliamente adoptado en la investigación de la ciencia de los alimentos, habiendo sido aplicada exitosamente para optimizar condiciones en varios procesos alimenticios.

Existen informes de autores extranjeros como: Mac Lellan, Barnard y Queale (1984), presentaron un estudio sobre un jugo de manzana carbonatada utilizando MSR.Milla, Algarbe y Tapia (2003), utilizaron la MSR en un néctar de mora pasteurizado .Pastor, Costell, Izquierdo y Durán (1996) Optimizing acceptability of a high fruit - low sugar peach nectar using aspartame and guar gum. Entre algunos informes de autores nacionales tenemos: Peñafiel, Morales y Salva (2006). Optimización con MSR en productos cárnicos. Pinto (1995). MSR con la optimización del proceso de clarificación de jugo de manzana.

En nuestro país existe una gran variedad de alimentos funcionales que no se explotan en su totalidad, se hace pues, necesario desarrollar productos en base a estos, siendo el camu camu la fruta elegida para el estudio, por tener la mayor cantidad de vitamina C.

La presente investigación se realizó con el fin de aplicar la Superficie de Respuesta en el desarrollo de un néctar de camu camu y su importancia radica en el empleo de una nueva técnica estadística que utiliza menor cantidad de pruebas experimentales, menores costos de investigación y producción para maximizar su aprobación sensorial facilitando la labor de los investigadores en la industria de los alimentos. Las limitaciones fueron pocas lográndose concluir el trabajo de investigación.

El objetivo general fue: "Optimización de un Néctar de Camu Camu (Myrciaria dubia) utilizando Métodología de Superficie de Respuesta.

ng. Freddy Bernabé Luza Mg. Sc., Profesor invitado perteneciente al Laboratorio de Tecnología de Alimentos de la Escuela Profesional de Ingenieria Alimentaria de la Universidad Nacional Federico Villareal 


\section{MATERIALES Y MÉTODOS}

\section{Elaboración del néctar}

En general se realizará el siguiente procedimiento: materia prima, lavado, pulpeado, refinado, dilución, adición de aditivos, homogenización, pasteurización, envasado y enfriado. Las muestras se almacenan para sus posteriores análisis.

\section{Evaluación sensorial}

Se emplean pruebas de medición del grado de satisfacción, para saber, cuanto les gusta o disgusta un producto Anzaldúa -Morales (1994). Se empleará una escala hedónica verbal de nueve puntos, ejecutadas por un panel de nueve jueces semientrenados. Esta prueba se efectuara en una hoja de calificación para una comparación pareada simple - diferencia para Análisis Discriminativo para determinar diferencia. (Ureña y D’Arrigo, 1999) La prueba de estabilidad se efectuará con un panel de 13 jueces semi entrenados con dos repeticiones por cada juez a un nivel de $\alpha=0,05$.

\section{Diseño experimental}

\section{Etapa de Screening}

El objetivo de esta etapa es observar los efectos que producen las variables (Ayala y Pardo ,1995).
El diseño utilizado es el diseño factorial $2^{k}$ con puntos centrales para 9 tratamientos que estime la existencia de curvatura (Millan et al., 2003 y Pinto, 1996).

Las variables consideradas fueron: $X_{1}=$ sacarosa $y$ $\mathrm{X}_{2}$ agua. Ver la Tabla 1.

Se decide una región de exploración con niveles para la sacarosa de $(52 \mathrm{~g}-72 \mathrm{~g})$ y para el agua de $(210 \mathrm{~g}-310 \mathrm{~g})$.La fórmula de cambio de variable es:

$$
\text { Sacarosa } X_{1}=\underline{E}_{1}-62 \text { Agua } X_{2}=\underline{E}_{2} \frac{-260}{50}
$$

\section{Etapa II: Escalamiento}

Se aplicó el proceso iterativo del método de máxima pendiente en ascenso, con un diseño $2^{2}$ con puntos centrales, para acceder a las cercanías del óptimo, en la dirección donde el crecimiento de la respuesta es máxima (Montgomery y Runger, 1996). Ver Tabla 2.

Se decide una región de exploración con niveles para la sacarosa de $(77 \mathrm{~g}-87 \mathrm{~g})$ y para el agua de $(280 \mathrm{~g}-320 \mathrm{~g})$. La ecuación de cambio de variable es:

Sacarosa $X_{1}=\underline{E}_{1}-82$; Agua $X_{2}=\underline{E}_{2}-320$ (2)

Tabla 1. Variables independientes y niveles para la etapa de screening

\begin{tabular}{lccccc}
\hline \multirow{2}{*}{ Variables } & \multicolumn{2}{c}{ Símbolo } & \multicolumn{3}{c}{ Niveles } \\
\cline { 2 - 6 } & Codificadas & Naturales & -1 & 0 & 1 \\
\hline Sacarosa & $\mathrm{x}_{1}$ & $\mathrm{X}_{1}$ & 52 & 62 & 72 \\
Agua & $\mathrm{x}_{2}$ & $\mathrm{X}_{2}$ & 210 & 260 & 310 \\
\hline
\end{tabular}

Tabla 2. Variables independientes y niveles para el segundo diseño experimental en la etapa de escalamiento

\begin{tabular}{lccrrr}
\hline \multicolumn{1}{c}{ Variables } & \multicolumn{2}{c}{ Símbolo } & \multicolumn{3}{c}{ Niveles } \\
\hline & Codificadas & Naturales & -1 & 0 & 1 \\
\hline Sacarosa & $\mathrm{X}_{1}$ & $\mathrm{X}_{1}$ & 77 & 82 & 87 \\
Agua & $\mathrm{X}_{2}$ & $\mathrm{X}_{2}$ & 280 & 300 & 320 \\
\hline
\end{tabular}


Etapa III : Optimización final. El diseño para modelar la superficie de respuesta de segundo orden fue un diseño central compuesto rotable según: (Montgomery,1991), (Mullen y Ennis,1979) y (Pastor et al. ,1996). Para $\mathrm{K}=2$ variables, consta de 4 puntos factoriales, 4 puntos axiales y 5 puntos centrales dando un total de 13 puntos experimentales. Para los factores y la porción factorial de $2^{2}=4$ puntos, el valor de $\alpha=(4)^{1 / 4}=$ 1,414 para lograr la invariabilidad ante el giro. El diseño se obtuvo del programa Minitab. Los datos se muestran en la siguiente Tabla 3:

Tabla 3. Diseño compuesto central

\begin{tabular}{llll}
\hline \multicolumn{2}{l}{ Variables codificadas } & \multicolumn{2}{l}{ Variables naturales } \\
\hline $\mathbf{x}_{1}$ & $\mathbf{X}_{2}$ & $\mathbf{X}_{1}$ & $\mathbf{X}_{2}$ \\
\hline+1 & +1 & 77 & 280 \\
+1 & -1 & 87 & 280 \\
-1 & +1 & 77 & 320 \\
-1 & -1 & 87 & 320 \\
$\sqrt{ } 2$ & 0 & 74,9289 & 300 \\
$-\sqrt{ } 2$ & 0 & 89,0711 & 300 \\
0 & $\sqrt{ } 2$ & 82 & 271,716 \\
0 & $-\sqrt{ } 2$ & 82 & 328,284 \\
0 & 0 & 82 & 300 \\
0 & 0 & 82 & 300 \\
0 & 0 & 82 & 300 \\
0 & 0 & 82 & 300 \\
0 & 0 & 82 & 300 \\
& & & \\
\hline
\end{tabular}

Los valores promedios de aceptación global serán sometidos a un análisis de regresión múltiple por el método de mínimos cuadrados y ajustados a un modelo de segundo orden incluyendo la curvatura e interacción para modelar la superficie.

La ecuación polinomial de segundo orden es:

$$
\begin{aligned}
& Y=\beta_{0}+\sum_{i=1}^{k} \beta_{i} X_{i}+\sum_{i=1}^{k} \beta_{i i} X_{i}^{2}+\sum_{i} \\
& \sum_{j} \beta_{i j} X_{i} X_{j}+\epsilon
\end{aligned}
$$

Donde: $\quad Y=$ Aceptación global,$\beta_{0}=$ Término constante, $\beta_{i}, \beta_{i i}=$ Coeficientes de regresión lineal y cuadrática, $X_{1}, X_{2}=$ Concentración de sacarosa y agua respectivamente.

Determinación de los niveles óptimos de las variables.

A fin de obtener una aproximación más analítica, los modelos obtenidos se expresaran en notación matricial:

$$
y=\beta_{0}+x^{\prime} b+x^{\prime} B x
$$

$$
\begin{aligned}
& X=\left(\begin{array}{l}
X 1 \\
X 2 \\
X k
\end{array}\right) \quad b=\left(\begin{array}{c}
\beta 1 \\
\beta 2 \\
\beta k
\end{array}\right) \\
& B=\left(\begin{array}{rrr}
\beta 11, & \beta 12 / 2 & \beta 1 \mathrm{k} / 2 \\
& \beta 22 & \beta 2 \mathrm{k} / 2 \\
& \beta \mathrm{kk}
\end{array}\right)
\end{aligned}
$$

Donde $\beta_{\circ}$ b y $B$ constituyen las estimaciones del intercepto, coeficientes lineales y cuadráticos respectivamente y $\mathrm{X}$ la matriz de las variables independientes del diseño. A partir de la expresión matricial del modelo de superficie se generó una expresión para la localización respecto a x. (Millan y Ostojich, 2005), (Montgomery, 1991) y (Reyes y Jiménez, 2006). Se halló los mejores niveles de las variables sacarosa y agua que obtuvieron la más alta puntuación al reemplazar en la ecuación:

La derivada de $\mathrm{y}$ con respecto al vector $\mathrm{x}$ igualada a 0 es :

$$
\underline{\partial \hat{y}}=b+2 B x=0
$$

$\partial x$

El punto estacionario es la solución de la ecuación:

$$
X_{O}=-1 / 2 B^{-1} b
$$

\section{La respuesta predicha}

La mejor puntuación de aceptabilidad global predicha se calcula con la ecuación:

$$
\text { ŷo }=\beta o+1 / 2 X o b
$$




\section{RESULTADOS Y DISCUSIONES}

\section{Etapa I: Screening}

Se obtuvo el modelo ajustado de primer orden en las variables codificadas siguiente:

$\hat{y}=5,5000+1,0000 x_{1}+0,0000 x_{2}+0,50000 x_{1} x_{2}(8)$

Donde $=$ el puntaje de aceptación global $\mathrm{y} \mathrm{x}_{1}, \mathrm{x}_{2}$ sacarosa y agua respectivamente.

El modelo es significativo a un nivel de $\alpha=0,05$ que implica que al menos una variable contribuye significativamente a la regresión. Las pruebas de significación para la interacción de los efectos y la prueba de significación para la curvatura también no fueron significativas. El modelo de la ecuación 8 ajusta adecuadamente los datos experimentales y pasar a la segunda etapa de escalamiento. Los coeficientes a un nivel de a $=0,05$ la variable sacarosa indica que si influye significativamente en el puntaje de aceptación global, la variable agua y la interacción no influyen significativamente.

\section{Etapa II: Escalamiento}

Se aplicó el proceso iterativo del método de máxima pendiente en ascenso al polinomio para acceder a las cercanías del óptimo. Se decide usar la sacarosa para un incremento en las variables naturales. El primer punto en la pendiente de máximo ascenso, fueron las variables $\left(x_{1}=62\right)$, $\left(x_{2}=260\right)$. Se observó un incremento en la puntuación hasta el quinto valor, a partir del sexto valor se observa un decremento en la respuesta que según Montgomery (1991), se debe ajustar otro modelo de primer orden en la cercanía de los valores $x_{1}=82 ; x_{2}=300$. Las variables codificadas son:

$$
X_{1}=\underline{E}_{1} \frac{-82}{5} ; X_{2}=\underline{E}_{2} \frac{-300}{20}
$$

El segundo modelo ajustado de primer orden en las variables codificadas es:

$=6,5000-1,0000 x_{1}-1,0000 x_{2}-1,5000 x_{1} x_{2}$

Tabla 4. Análisis de varianza de los efectos de las variables para la aceptación

\begin{tabular}{llllll}
\hline \multicolumn{1}{c}{$\begin{array}{c}\text { Fuente de } \\
\text { variabilidad }\end{array}$} & Grado de libertad & $\begin{array}{c}\text { Suma de } \\
\text { cuadrados }\end{array}$ & Cuadrado medio & F & P \\
\hline Efectos principales & 2 & 4,00000 & 2,0000 & 10,00 & 0,028 \\
Interacciones & 1 & 1,00000 & 1,0000 & 5,00 & 0,089 \\
Curvatura & 1 & 1,08889 & 1,0889 & 5,44 & 0,080 \\
Error residual & 4 & 0,80000 & 0,2000 & & \\
Error puro & 4 & 0,80000 & 0,2000 & & \\
Total & 8 & 6,88889 & & & \\
\hline
\end{tabular}

Tabla 5. Análisis de varianza para el segundo modelo de las variables

\begin{tabular}{|l|l|c|c|c|c|}
\hline \multicolumn{1}{|c|}{ X2 } & Grados de libertad & $\begin{array}{c}\text { Suma de } \\
\text { cuadrados }\end{array}$ & Cuadrado medio & F & P \\
\hline Efectos principales & 2 & 8,000 & 4,000 & 5,00 & 0,082 \\
Interacciones & 1 & 9,000 & 9,000 & 11,25 & 0,028 \\
Curvatura & 1 & 9,800 & 9,800 & 12,25 & 0,025 \\
Error residual & 4 & 3,200 & 0,800 & & \\
Error puro & 4 & 3,200 & 0,800 & & \\
Total & 8 & 30,000 & & & \\
\hline
\end{tabular}


A un nivel de confianza del $95 \%$, el modelo no es adecuado. Las pruebas de significación para la interacción y de curvatura de los efectos fueron significativas e indican la falta de ajuste en este modelo de primer orden y se debe emplear un modelo de segundo orden.

\section{Etapa III : Optimización final}

El diseño para modelar la superficie de respuesta de segundo orden fue un diseño central compuesto rotable según: (Montgomery,1991), (Mullen y Ennis, 1979) y (Pastor et al. ,1996). Para K $=2$ variables, consta de 4 puntos factoriales, 4 puntos axiales en los ejes coordenados (a una distancia $\alpha=1,414)$ y 5 puntos centrales dando un total de 13 puntos experimentales. El diseño se obtuvo del programa computacional. Minitab. Ver la Tabla 6.

Tabla 6. Diseño compuesto central

\begin{tabular}{cccc}
\hline \multicolumn{2}{c}{ Variables codificadas } & \multicolumn{2}{c}{ Variables naturales } \\
\hline $\mathrm{X}_{1}$ & $\mathrm{X}_{2}$ & $\mathrm{X}_{1}$ & $\mathrm{X}_{2}$ \\
\hline+1 & +1 & 77 & 280 \\
+1 & -1 & 87 & 280 \\
-1 & +1 & 77 & 320 \\
-1 & -1 & 87 & 320 \\
$\sqrt{2}$ & 0 & 74,9289 & 300 \\
$-\sqrt{2}$ & 0 & 89,0711 & 300 \\
0 & $\sqrt{2}$ & 82 & 271,716 \\
0 & $-\sqrt{2}$ & 82 & 328,284 \\
0 & 0 & 82 & 300 \\
0 & 0 & 82 & 300 \\
0 & 0 & 82 & 300 \\
0 & 0 & 82 & 300 \\
0 & 0 & 82 & 300 \\
& & & \\
\cline { 2 - 3 } & & &
\end{tabular}

Los valores promedios de aceptación global fueron sometidos a un análisis de regresión múltiple por el método de mínimos cuadrados y ajustados a un modelo de segundo orden incluyendo la curvatura e interacción para modelar la superficie. El diseño se obtuvo utilizando el programa computacional Minitab. La ecuación polinomial de segundo orden fue:

$\hat{y}=8,8000-0,5000 x_{1}-1,0303 x_{2}-0,8375 x_{1}^{2}-$ $1,5875 x_{2}^{2}-1,5000 x_{1}, x_{2}$
Donde: $\hat{y}=$ puntaje de aceptación global; $x_{1}, x_{2}=$ sacarosa y agua respectivamente.

\section{Adecuación del modelo matemático de segundo grado}

El modelo de regresión es significativo a un nivel de confianza de $95 \%$. Los términos cuadráticos del modelo son significativos, lo que revela la identificación de un punto de aceptación global óptimo, (Millan et al., 2003). La falta de ajuste no es significativo a un nivel de confianza de $95 \%$. El coeficiente $\left(R^{2}\right)$ explica que un $93,1 \%$ de la variabilidad de los datos es por el modelo. La prueba de Durbin - Watson de los residuales es 1,626 que indica que no existe una autocorrelación seria de los residuales. Ver Tabla 7.

(Villar et al.,2007) señala, que los coeficientes son estadísticamente confiables si los $\mathrm{R}^{2}$ son mayores al $90 \%$ y el coeficiente de Durbin - Watson es cercano a 2. Ver Tabla 8.

De la tabla 8 se puede apreciar que los coeficientes de las variables en estudio: $x_{1}, x_{2}, x_{1}^{2}, x_{2}^{2}, x_{1} x_{2}$; son significativas al nivel de significancia $(\alpha=0,05)$.

\section{Determinación de los niveles óptimos de las variables}

Al reemplazar en la ecuación: $\quad x=-1 / 2 \mathrm{~B}^{-1} \mathrm{~b}$

Se determinó el punto estacionario en términos de las variables codificadas, esto es: $x_{1}=-0,4082$ y $\quad x_{2}=0,0613$. Reemplazando en la fórmula de cambio de variable (2). En términos de las variables naturales el punto estacionario es: para la sacarosa $x_{1}=79,95 \mathrm{~g}$ y para el agua $x_{2}=301,22 \mathrm{~g}$.

Determinación de la aceptación global predicha Desarrollando la ecuación:

$\hat{y}=\beta_{0}+1 / 2 X^{1}{ }_{o} b$

La respuesta predicha en el punto estacionario es: $\hat{y}=8,86$.

\section{CONCLUSIONES}

Los niveles óptimos de las variables fueron: Sacarosa : 79,95 g, Agua : 301,22 g

La formulación final del néctar de camu camu fue :

Agua $=66,60 \%$, Sacarosa $=17,68 \%$, Zumo de camu camu $=15,47 \%$, Carboxilmetilcelulosa $=$ $0,1 \%$, Sorbato de potasio $=0,15 \%$.

El mejor puntaje de aceptabilidad fue de 8,86 puntos, de un total de nueve puntos. 
Tabla 7. Análisis de varianza de la regresión para la aceptación global

\begin{tabular}{lccccc}
\hline $\begin{array}{c}\text { Fuente de } \\
\text { variabilidad }\end{array}$ & $\begin{array}{c}\text { Grados de } \\
\text { libertad }\end{array}$ & $\begin{array}{c}\text { Suma de } \\
\text { cuadrados }\end{array}$ & Cuadrado medio & $\mathbf{F}_{\mathrm{c}}$ & $\mathbf{P}$ \\
\hline Regresión & 5 & 39,8369 & 7,9674 & 19,02 & 0,001 \\
Lineal & 2 & 10,4926 & 5,2463 & 12,52 & 0,005 \\
Cuadrático & 2 & 20,3442 & 10,1721 & 24,28 & 0,001 \\
Interacción & 1 & 9,000 & 9,000 & 21,48 & 0,002 \\
Error residual & 7 & 2,9324 & 0,4189 & & \\
Falta de ajuste & 3 & 2,1324 & 0,7108 & 3,55 & 0,126 \\
Error puro & 4 & 0,8000 & 0,2000 & & \\
Total & 12 & 42,7692 & & & \\
\hline
\end{tabular}

$R^{2}=93,1 \% \quad R^{2}$ adj. $=88,2 \% \quad$ Durbin - Watson $=1,626$

Tabla 8. Análisis de significancia de los coeficientes de la ecuación cuadrática

\begin{tabular}{lcccc}
\hline $\begin{array}{c}\text { Término del } \\
\text { modelo }\end{array}$ & Coeficiente & $\begin{array}{c}\text { Error del } \\
\text { coeficiente }\end{array}$ & $\mathbf{T}$ & $\mathbf{P}$ \\
\hline Constante & 8,8000 & 0,2895 & 30,402 & 0,000 \\
$\mathrm{x}_{1}$ & $-0,5000$ & 0,2288 & $-2,185$ & 0,022 \\
$\mathrm{x}_{2}{ }^{2}$ & $-1,0303$ & 0,2288 & $-4,503$ & 0,022 \\
$\mathrm{x}_{2}^{2}$ & $-0,8375$ & 0,2454 & $-3,413$ & 0,016 \\
$\mathrm{x}_{2}{ }^{2}$ & $-1,5875$ & 0,2454 & $-6,469$ & 0,001 \\
$\mathrm{x}_{1}, \mathrm{x}_{2}$ & $-1,5000$ & 0,3236 & $-4,635$ & 0,002 \\
\hline
\end{tabular}

Figura 1. Contorno de aceptación vs. sacarosa, agua.

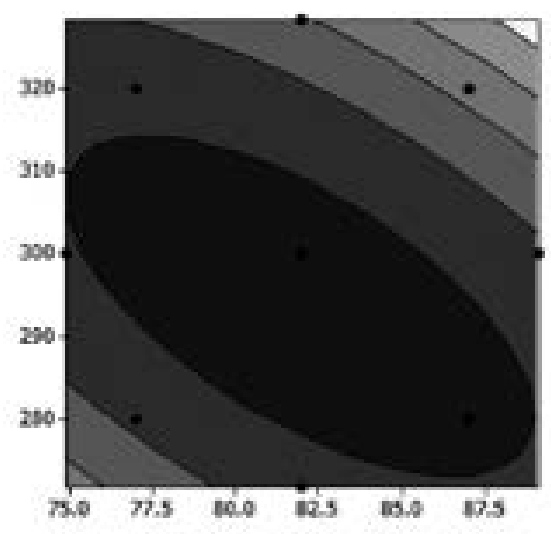

Fuente: Elaboración propia.
Figura 2. Siperficie de respuesta: aceptación vs. sacarosa, agua.
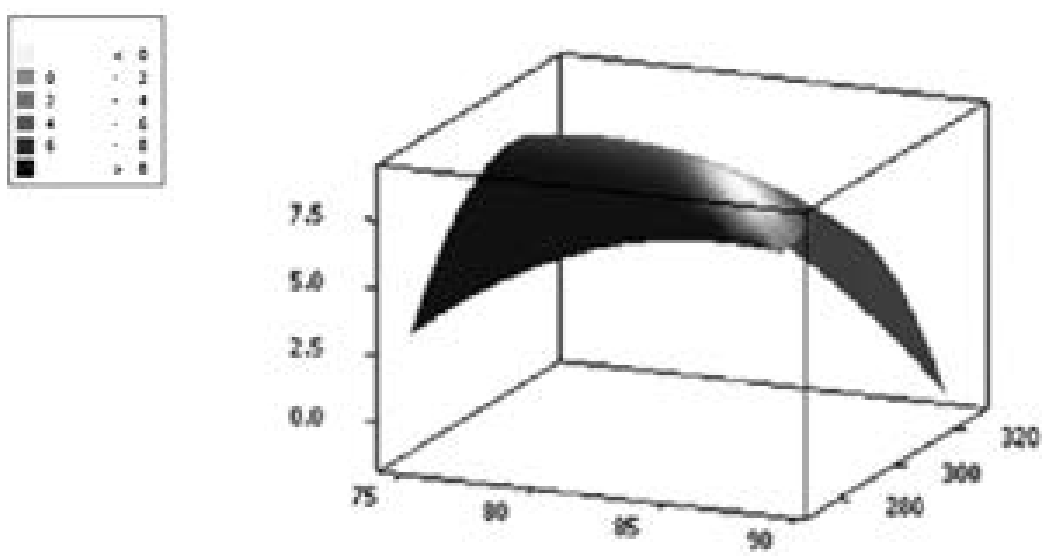

Fuente: Elaboración propia. 
El néctar tuvo un $\mathrm{pH}=3,4$, indica la baja acidez que posee el zumo. El tiempo de vida útil del néctar fue de 80 días.

\section{REFERENCIAS BIBLIOGRÁFICAS}

[1] Anzaldúa - Morales, A. (1994). La evaluación sensorial de los alimentos en la teoría y práctica. España: Acribia.

[2] Ayala, J. y Pardo, R. (1995) Optimización por diseños experimentales con aplicaciones en Ingeniería. Lima: CONCYTEC.

[3] Mc. Lellan M.; Quale, D. y Barnard, J. (1984). Sensory analysis of carbonated apple juice using response surface methodology. Journal of Food Science, 49, 1596 - 1600.

[4] Millán,F. y Ostojich, Z. (2005). Aplicación de un diseño rotable en el modelado empirico de la deshidratación osmótica en frutas. Universidad Simón Bolívar: Venezuela.En http://www.scielo. org.ue/scielo.php? Pid=50378/8442005 0010000 $10 \&$ script $=$ sci- art text. Visitado el 11/10/2009.

[5] Millán, F.; Tapia, M. y Algarbe, M. (2003). Uso de la metodología de superficie de respuesta y la programación lineal para el desarrollo de un néctar de mora pasteurizado. Interciencia, 28, 646-649.

[6] Montgomery, D. y Runger, G. (1996). Probabilidad y estadística aplicada a la ingeniería. México: McGraw - Hill.

[7] Montgomery, D. (1991). Métodos y diseños de superficie de respuesta: Diseños y análisis de experimentos. España: Iberoamericana.
[8] Mullen, K. y Ennis, D. (1979). Rotatable designs in product development. Journal of Food Technology. (pp 74-80).

[9] Pastor, M.V; Costell, E.; Izquierdo, L. \& Durán, L. (1996). Optimizing acceptability of a high fruit - low sugar peach nectar using aspartame and guar gum. Journal of Food Science, 61, 4, 852-855.

[10] Peñafiel; C.E.; Morales, E. y Salva, B. (2006) Optimización por aplicación del método de superficie de respuesta en productos cárnicos. La Molina: Agraria.

[11] Pinto, J. (1995). Aplicación de la metodología de superficie de respuesta en la optimización del proceso de clarificación de jugo de manzana. Tesis para obtener el título de Ingeniero en Industrias Alimentarias. Lima: Universidad Agraria La Molina.

[12] Reyes, P. y Jiménez, E. (2006). Superficies de respuesta de segundo orden. En: http:// www.icicm.com/files/modelodesegundoorden. doc. Doc. Resumen del primer capítulo de investigación de mercados. Visitado el 5/3/2009.

[13] Ureña, M. y D. Arrigo, M. (2003). Evaluación sensorial de los alimentos. Lima : Editorial Agraria.

[14] Villar, J.M. (2006). Estadística 2: Chequeo del modelo de regresión múltiple. La Coruña: Universidad de la Coruña. En: http://dm.udc,es/ asignaturas/estadística2/estadística_2htm. Visitado el 24/07/2009. 